\title{
Persistence of the spacing effect in incidental free recall: The effect of external list comparisons and intertask correlations
}

\author{
THOMAS D. JENSEN and JOEL S. FREUND \\ University of Arkansas, Fayetteville, Arkansas 72701
}

\begin{abstract}
The attenuation-of-attention explanation of the spacing effect was tested, using an incidental learning paradigm. The incidental task was one in which subjects rated each item on either one or two scales. When two scales were used, all repeated items (massed and distributed) were rated on both scales. In Experiment 1, each point on the rating scale was labeled with an exemplar, and subjects were asked to compare each list item with the given exemplars. In Experiment 2, the correlation between the two scales was systematically varied. Neither manipulation reduced the magnitude of the spacing effect.
\end{abstract}

According to the total-time hypothesis, the amount learned is a direct function of the study time regardless of the distribution of that time (e.g., Cooper \& Pantle, 1969). However, it has repeatedly been demonstrated that distributed presentation of items within a list results in better recall than does massed presentation (see Hintzman, 1974, for a review). Although a number of theoretical explanations for the spacing effect have been proposed, the experiments reported here focus primarily on the attenuation-of-attention hypothesis.

According to this hypothesis, the spacing effect results because individuals do not process the second presentation of massed (MP) items to the same extent as the second presentation of distributed (DP) items. It is further assumed that this differential processing is a result of a voluntary shift in attention on the part of the subjects. Following this reasoning, Hintzman (1974) argued that instructional manipulations designed to increase attention to the MP items should reduce the magnitude of the effect. However, informing subjects that they would be paid for recalling certain MP or DP items failed to reduce the effect; rather, the monetary incentives resulted in increased recall for both types of items. Furthermore, instructions to pay attention to and to rehearse only the particular item presented have failed to reduce the effect (Underwood, 1969).

In a further test of this hypothesis, Shaughnessy (1976) used an incidental learning paradigm in which the cover task involved rating the words for imagery or frequency. The task was designed to induce a deep, or semantic, level of processing for each item (see Craik \& Lockhart, 1972). In two experiments (1 and 3), subjects either rated all items on the same scale or rated repetitions of the items on different scales. Shaughnessy hypothesized that rating the two occurrences of MP

Address reprint requests to J. S. Freund, Department of Psychology, University of Arkansas, Fayetteville, Arkansas 72701. items on different scales would force subjects to attend equally to both occurrences and, thus, would reduce the spacing effect; it did not reduce the spacing effect.

Although Shaughnessy's (1976) results present prima facie evidence against a voluntary attenuation-ofattention hypothesis, it is possible that they simply exemplify the difficulty of controlling the subjects' volitional processing strategies. Shaughnessy suggested that, although the rating instructions may have increased processing of repeated items, this increase may have been offset by an alteration in the pattern of displaced rehearsals, resulting in no change in the spacing effect. That is, when asked to rate items on a subjective scale, subjects may compare each to-be-rated item with previously rated items (Postman, 1975). Because DP items are more likely to serve as comparisons for subsequent list items (Rundus, 1971), use of rating scales would not necessarily eliminate the differential processing of MP and DP items.

\section{EXPERIMENT 1}

Experiment 1 was designed to test Shaughnessy's (1976) hypothesis by preventing the within-list rehearsals resulting from the use of rating scales. To prevent these comparisons, subjects were given an exemplar for each point on the scale and instructed to compare each to-beremembered item with the exemplars. If the spacing effect found following the use of rating tasks is due to increased within-list comparisons that offset the advantage gained by increased processing, reducing these rehearsals should reduce the effect.

\section{Method}

The experiment included two incidental learning groups and an intentional control group. Subjects in the intentional condition were given free recall instructions. Subjects in the single-scale condition rated each word on a single characteristic (frequency or imagery). Subjects in the two-scale group used both scales. Thus, for words occurring twice, subjects rated the first occurrence of the word on one scale and the second presentation on 
the other scale. Subjects in the incidental conditions were instructed to rate each word by comparing it with the examples printed on the scale.

Materials. The study list consisted of 12 words in each category [once-presented (OP), MP, and DP] ; MP and DP items were presented only twice. A primacy buffer consisted of two OP items, one MP item, and one DP item; a recency buffer consisted of four OP items. All DP items were separated by a lag of four, five, or six items. The main body of the study list was divided into quarters, such that each quarter contained three OP, three MP, and three DP items.

The 36 words used in the experiment were selected from the Paivio, Yuille, and Madigan (1968) norms. Nine words fell within each cell of the factorial combinations of frequency (low, high) and imagery (low, high). Low-frequency words had ThorndikeLorge frequencies of 20 or less per million; high-frequency words had A or AA ratings. Low-imagery words were rated 2.00 or less; high-imagery words had ratings of 6.00 or more. The words were divided into three sets by block randomization, with three words from each frequency-imagery combination occurring in each set. Each set was then assigned to be OP, MP, or DP items. The words within each set were then randomly assigned to positions within the list, with the restriction that equal numbers of words from each frequency-imagery combination occur within each fourth of the list. Six different study lists were constructed. Across all lists, each set occurred equally often as MP, DP, and OP items.

The rating sheets given to the subjects in the single-scale conditions contained 70 numbered blanks, with a 3-point scale at the top of the page. Each point on the scale was labeled with an appropriate example, representing low, medium, or high frequency or imagery. The three example words for the imagery scale were "explanation," "genius," and "barrel"; those for the frequency scale were "panorama," "inhabitant," and "air." In the two-task condition, both scales appeared at the top of the page. Half of the OP items were rated on frequency and the other half on imagery. Half of the twice-presented items under each schedule (MP or DP) were rated on one scale first and on the other second; for the other half, this procedure was reversed.

Subjects. Fourteen subjects were assigned to each of the three conditions and received partial credit toward a course requirement. Subjects were run individually and were assigned to conditions and study lists according to a block-randomized schedule. Seven psychology graduate students served as experimenters.

Procedure. Subjects in the incidental conditions were told that the experiment dealt with how people rated words on frequency, imagery, or both, depending on the condition. The rating scale(s) was (were) then explained, and subjects were told that, although some words would occur more than once, they should try to rate each word as if it were new. Subjects in the intentional condition were given standard free recall instructions. The study list was presented orally at a $5 \mathrm{sec}$ rate, after which all subjects were given a self-paced free recall test.

\section{Results}

The mean numbers of items recalled for OP, MP, and DP items for each of the three instructional conditions are presented in Table 1. An ANOVA of the number of items recalled revealed a significant effect for type of item $[\mathrm{F}(2,78)=24.70, \mathrm{MSe}=2.07]$. The DP items were recalled better than MP and OP items. The main effect of number of rating scales and the interaction of items and scales were not significant $(\mathrm{Fs}<1)$.

As a more sensitive test of the spacing effect, the twicepresented words were analyzed separately. Only the main effect of type of item was significant $[F(1,39)=$ $37.78, \mathrm{MSe}=1.82]$; more $\mathrm{DP}$ items were recalled than MP items (all other Fs $<1$ ). The use of specific example
Table 1

Mean Number of Words Recalled as a Function of Item Type and Condition

\begin{tabular}{lrrrrrrrr}
\hline & \multicolumn{2}{c}{ OP Items } & & \multicolumn{2}{c}{ MP Items } & & \multicolumn{2}{c}{ DP Items } \\
\cline { 2 - 3 } Condition & Mean & SD & & Mean & SD & & Mean & SD \\
\hline Intentional & 1.93 & 1.68 & & 2.07 & 1.38 & & 4.07 & 2.27 \\
Incidental & & & & & & \\
$\quad$ One Scale & 2.00 & 1.66 & & 1.79 & 1.48 & & 3.85 & 1.92 \\
$\quad$ Two Scales & 1.71 & .99 & & 2.36 & 1.39 & & 3.71 & 1.64 \\
\hline
\end{tabular}

Note-Maximum recall per cell $=12$.

words to reduce the within-list comparisons failed to reduce the spacing effect.

To determine if recall was affected by the type of rating scale, the data from the single-scale conditions were analyzed. More DP than OP and MP items were recalled $[F(2,24)=7.20, \mathrm{MSe}=2.39]$. No other effects approached significance. The type of rating scale (frequency, imagery) did not affect subsequent recall.

\section{Discussion}

We expected that the use of an incidental learning task preventing displaced rehearsals would reduce the spacing effect. The failure of this manipulation lends further support to a nonvolitional processing deficit. explanation of the spacing effect (Shaughnessy, 1976). However, as Shaughnessy noted, attempts to reduce the spacing effect, assuming the processing deficit is voluntary, may not have controlled those voluntary processes or may not have been used in conjunction with other variables that contribute to the superiority of DP item recall.

One assumption underlying Shaughnessy's (1976) experiment and Experiment 1 was that rating items on two different scales would increase the number of available recall cues. However, if the scales chosen were correlated, subjects may not have contacted different cues with each scale, and any resulting increase in recall would be minimal. Research by Battig and Einstein (1977) and Klein and Saltz (1976) supports this interpretation. Klein and Saltz found an inverse relationship between recall and the correlation between two rating scales. Thus, Shaughnessy's (1976) and our failure to reduce the spacing effect may be due to the high correlation between the scales.

In Experiment 2, subjects were asked to rate words on either one or two scales. In the two-scale conditions, the correlation between the two scales was varied. If each rating of a word contacts potential retrieval cues, then the number of potential retrieval cues (and, therefore, probability of recall) should vary inversely with the correlation between the two scales. Furthermore, if the poor recall of MP items results from the fact that fewer recall cues are available with MP than with DP items, using two uncorrelated rating scales should increase the recall of MP items more than recall of DP items.

\section{EXPERIMENT 2}

Five independent groups were used in Experiment 2. The intentional group was given standard free recall instructions and did not perform any ratings. The other four conditions involved incidental learning. In the singlescale condition, subjects were required to rate each item on one of four semantic dimensions (pleasant-unpleasant, good-bad, hard-soft, vivid-dull). It was expected that the 
spacing effect produced in this condition would not differ from that in the intentional condition.

The remaining incidental conditions were two-scale conditions, with the correlations between the two scales varying from low (vivid-dull, hard-soft) to moderate (good-bad, hard-soft) to high (pleasant-unpleasant, goodbad). If the attenuation-of-attention analysis of the spacing effect is correct, the size of the effect should vary inversely with the correlation between the two scales.

\section{Method}

Interscale correlations. The correlations between the scales were obtained using the method employed by Klein and Saltz (1976). Twenty subjects who did not serve in the main experiment rated all 24 possible pairs of four bipolar dimensions. The dimensions rated were selected because of their varying intercorrelations, as reported by Wilcox and Pollio (Note 1). The correlations calculated using the Klein and Saltz method were $.95, .50$, and -.18 for good-bad with pleasant-unpleasant, goodbad with hard-soft, and hard-soft with vivid-dull, respectively. The correlations reported by Wilcox and Pollio were $.92, .52$, and .09 for the same pairs of dimensions.

Subjects. The 48 subjects assigned to each of the five conditions were students who received partial credit toward a course requirement. Subjects were assigned to conditions according to a block-randomized schedule and were run in groups ranging in size from one to eight persons.

List construction. The study list presented to each subject was made up of 18 items: $6 \mathrm{OP}, 6 \mathrm{MP}$, and $6 \mathrm{DP}$. The main body of the study list was divided in half, such that each half contained three items of each type. The primacy buffer consisted of two OP, one MP, and one DP items; the recency buffer consisted of four OP items. All DP items were separated by a lag of four, five, or six items. All of the words were selected from Wilcox and Pollio (Note 1) to match the correlations that had already been determined between the various scales. The correlations for the words used were $.95, .57$, and -.01 for goodbad with pleasant-unpleasant, good-bad with hard-soft, and hardsoft with vivid-dull, respectively.

The 18 items composing the main body of the study list were randomly divided into three sets, which were then assigned to be OP, MP, or DP items. Two additional forms of the list were constructed so that, across forms, all words served as OP, MP, and DP items. All three lists were presented to an equal number of subjects within each condition.

The rating sheet given to each subject consisted of 40 numbered dimensions. The scale that subjects were to employ was printed beside the corresponding number on the list. The scales consisted of the two poles of the dimension (e.g., good-bad), and subjects were to circle their response. Half of the OP items in the two-scale condition were rated on one scale, and half were rated on the other. Half of the MP and DP items were rated first on one scale and then on the other. One-fourth of the subjects in the single-task condition rated the words on each dimension.

Procedure. Subjects in the intentional condition were given standard free recall instructions. Subjects in the remaining four conditions were told: (1) that the experiment dealt with how people rate words on different scales, (2) that some words would occur more than once but they should rate each word as if it were new, and (3) to circle on the scale the response they felt was most appropriate for the corresponding word in the list. Subjects were verbally presented an example using a word and scale not used in the study. The study-list words were presented on a tape recorder at a $5-\mathrm{sec}$ rate, with each word in the study list spoken twice during the $5 \mathrm{sec}$ to ensure comprehension. After presentation of the study list, all subjects were given a self-paced free recall test.

\section{Results}

An ANOVA conducted on the recall data obtained from subjects in the single-scale condition produced a significant main effect of distribution (OP, MP, DP) $[\mathrm{F}(2,88)=18.54, \mathrm{MSe}=1.66]$; subjects recalled more $\mathrm{MP}$ and DP items than OP items $[\mathrm{F}(1,88)=22.62]$ and more DP than MP items $[F(1,88)=14.46]$. The main effect of type of scale and the Scale by Distribution interaction were not significant. As in Experiment 1, when only a single scale was used, the type of scale did not influence either total words recalled or the size of the MP-DP effect.

The mean numbers of words recalled and the standard deviations for each of the five conditions for OP, MP, and DP items are presented in Table 2. A 3 by 5 (distribution by groups) ANOVA on the number of words recalled revealed significant main effects for groups $[\mathrm{F}(4,235)=$ $6.49, \mathrm{MSe}=1.18]$ and distribution $[\mathrm{F}(2,470)=88.13$, $\mathrm{MSe}=1.35]$. The Groups by Distribution interaction was also significant $[\mathrm{F}(8,470)=2.52$, MSe $=1.35]$.

Further analyses investigating the Groups by Distribution interaction revealed a significant simple main effect indicating that subjects in the different conditions recalled differing numbers of OP items $[\mathrm{F}(4,235)=10.24, \mathrm{MSe}=$ 1.21]. Orthogonal comparisons revealed that intentional subjects recalled more DP items than subjects using either one or two scales $[F(1,235)=32.63]$. Subjects using a single scale recalled more OP items than did subjects using two scales $[F(1,235)=4.33]$, and subjects using highly correlated scales recalled more OP items than did subjects using poorly correlated scales $[\mathrm{F}(1,235)=3.43]$. Recall of OP items for subjects using moderately correlated scales did not differ from recall for subjects using highly and poorly correlated scales $(\mathrm{F}<1)$. The lower the correlation between scales (assuming for the singlescale group an $r=+1.00$ ) used for rating MP and DP items, the greater was the interference on recall of OP items. The groups did not differ on the number of MP and DP items recalled $[F(4,235)=2.05, \mathrm{MSe}=1.56$, and $\mathrm{F}(4,235)=1.69, \mathrm{MSe}=1.74$, respectively] .

Orthogonal planned comparisons on the main effect of groups revealed that subjects in the intentional group recalled more words than did subjects in the combined

Table 2

Mean Number of Words Recalled and Standard Deviations in Each Condition for OP, MP, and DP Items

\begin{tabular}{|c|c|c|c|c|c|c|}
\hline \multirow[b]{2}{*}{ Condition } & \multicolumn{2}{|c|}{ OP Items } & \multicolumn{2}{|c|}{ MP Items } & \multicolumn{2}{|c|}{ DP Items } \\
\hline & Mean & SD & Mean & SD & Mean & SD \\
\hline Intentional & 2.25 & 1.42 & 2.10 & 1.26 & 2.90 & 1.36 \\
\hline Single Scale & 1.52 & 1.01 & 2.10 & 1.31 & 3.10 & 1.46 \\
\hline \multicolumn{7}{|l|}{ Two Scales } \\
\hline High Correlation & 1.40 & 1.11 & 2.23 & 1.17 & 2.81 & 1.30 \\
\hline Moderate Correlation & 1.04 & .99 & 2.02 & 1.31 & 2.94 & 1.36 \\
\hline Low Correlation & .98 & .91 & 1.56 & 1.18 & 2.44 & 1.09 \\
\hline
\end{tabular}

Note-Maximum recall per cell $=6$. 
incidental groups $[\mathrm{F}(1,235)=10.40, \mathrm{MSe}=1.81]$. Subjects using only one scale recalled more words than did subjects using two scales $[\mathrm{F}(1,235)=5.65]$. Subjects using two highly correlated scales recalled more words than did subjects using two poorly correlated scales $[F(1,235)=9.39]$. Recall for the moderate condition did not differ from either the low or the high condition. Thus, recall decreased as a function of the number of scales, and as the magnitude of the correlation between scales decreased.

Planned comparisons conducted on the main effect of distribution revealed that subjects recalled more twicepresented items than OP items $[\mathrm{F}(1,470)=114.52]$ and more DP items than MP items $[\mathrm{F}(1,470)=61.68, \mathrm{MSe}=$ $1.35]$. Thus, from the above analyses, the attempt to reduce the MP-DP effect failed.

A more sensitive test for variation in the size of the spacing effect as a function of the strength of the correlation between scales was conducted by analyzing only the twice-presented items in the incidental conditions. The analysis revealed significant main effects of groups $[\mathrm{F}(3,188)=4.19, \mathrm{MSe}=1.70$, and item distribution $[\mathrm{F}(1,188)=43.67, \mathrm{MSe}=1.57]$. The Groups by Distribution interaction was not significant $(F<1)$. Regardless of the correlation between tasks, subjects recalled more DP items $($ mean $=2.823)$ than MP items (mean = 1.979). Comparisons revealed that subjects performing a single task and subjects performing highly correlated tasks recalled more words than did subjects performing poorly correlated tasks $[F(1,188)=7.66]$. No other significant differences were found.

\section{GENERAL DISCUSSION}

According to the attenuation-of-attention hypothesis, the spacing effect results from differential processing of MP and DP items, which is a consequence of voluntary shifts in attention. The results of the present experiments and those of Hintzman (1974), Shaughnessy (1976), and Underwood (1969) question at least the voluntary portion of this hypothesis. Both Hintzman and Underwood failed to reduce the size of the spacing effect through instructional manipulations, and Shaughnessy's attempt to control differential attention and differential processing was not successful.

Experiment 1 tested the hypothesis that Shaughnessy's (1976) manipulations failed because the subjective nature of the rating scale resulted in displaced rehearsals. Providing subjects with comparison words that were not in the list failed to reduce the size of the spacing effect.

In Experiment 2, the correlation between the two scales used for rating items was systematically varied, with the expectation that a low interscale correlation would result in approximately equal elaborative processing for MP and DP items and, thus, in a reduction of the spacing effect. This prediction was not supported; the spacing effect remained invariant across the levels of the interscale correlation. However, contrary to previous results (Klein \& Saltz, 1976), recall varied directly with the magnitude of the interscale correlation.

When combined with the results of previous investigations, the present results suggest that the spacing effect may not be due to a voluntary processing deficiency of MP items (Hintzman, 1974). However, some questions must be answered before a strong conclusion can be made. Because Experiment 1 did not include a manipulation check to determine if supplying examples would prevent within-list rehearsals, it is possible that the manipulation did not have the desired effect. This possibility should be examined. A second question concerns the failure to replicate the Klein and Saltz (1976) results in Experiment 2. Although the procedures used in the two studies were not identical, even when the two experiments are comparable (our MP items) the results were conflicting. Clearly, additional work must be done to examine this replication failure.

\section{REFERENCE NOTE}

1. Wilcox, R., \& Pollio, H. Semantic ratings for 630 words and some correlated associative properties (Tech. Rep. 3). Knoxville: University of Tennessee, Department of Psychology, 1966.

\section{REFERENCES}

Battig, W. F., \& Einstein, G. O. Evidence that broader processing facilitates delayed retention. Bulletin of the Psychonomic Society, 1977, 10, 28-30.

Cooper, E. H., \& Pantle, A. J. The total-time hypothesis in verbal learning. Psychological Bulletin, 1969, 68, 221-234.

Craik, F. I. M., \& Lockhart, R. S. Levels of processing: A framework for memory research. Journal of Verbal Learning and Verbal Behavior, 1972, 11, 671-684.

Hintzman, D. L. Theoretical implications of the spacing effect. In R. L. Solso (Ed.), Theories in cognitive psychology: The Loyola symposium. Potomac, Md: Erlbaum, 1974.

KLe in, F., \& SAltz, E. Specifying the mechanism in a levels-ofprocessing approach to memory. Journal of Experimental Psychology: Human Learning and Memory, 1976, 2, 671-679.

Paivio, A., Yuille, J. C., \& Madigan, S. A. Concreteness, imagery, and meaningfulness values for 925 nouns. Journal of Experimental Psychology Monograph, 1968, 76(1, Part 2), 1-25.

Postman, L. Verbal learning and memory. Annual Review of Psychology, 1975, 26, 291-335.

Rundus, D. Analysis of rehearsal processes in free recall. Journal of Experimental Psychology, 1971, 89, 63-77.

Shaughnessy, J. J. Persistence of the spacing effect in free recall under varying incidental learning conditions. Memory \& Cognition, 1976, 4, 369-377.

UNDERWOOD, B. J. Some correlates of item repetition in free recall learning. Journal of Verbal Learning and Verbal Behavior, $1969,8,83-94$.

(Received for publication June 23, 1981.) 